\title{
Motives of cheating among secondary students: the role of self-efficacy and peer influence
}

\author{
Wong Lok Yan Nora $\cdot$ Kaili Chen Zhang
}

Received: 1 September 2009/Revised: 18 May 2010/Accepted: 19 May 2010/Published online: 9 June 2010

(C) The Author(s) 2010. This article is published with open access at Springerlink.com

\begin{abstract}
A survey research study was conducted with a sample of 100 secondary students from a local secondary school about the motives of cheating. The primary focus of this study was the interplay among variables of self-efficacy, peer influence and cheating. The results showed that students with low self-efficacy were more likely to cheat than those who perceived themselves as efficacious. It was further found that peers played a significant role in discouraging cheating by expressing disapproval and informing teachers of dishonest behaviour.
\end{abstract}

Keywords Self-efficacy · Peer influence ·

Academic dishonesty $\cdot$ Cheating

Over the years, research on academic dishonesty has been dominated by attempts to examine two very practical aspects: the characteristics of cheaters and the effectiveness of deterrents to academic dishonesty. In exploring these research areas, the self-reporting type of questionnaire is most frequently utilised to quantitatively assess factors associated with cheating. Taylor et al. (2003) commented that "the existing literature, however, lacks in-depth, qualitative studies on academic dishonesty" (p. 403). Specifically, little has been conducted to qualitatively assess whether self-efficacy and peer influence affect the likelihood of students engaging in academic dishonesty.

The level of participants being studied was another major limitation of previous research. Most of the studies have focused on cheating found in tertiary education, often

W. L. Y. Nora $\cdot$ K. C. Zhang $(\bowtie)$

Faculty of Education, The University of Hong Kong, Pokfulam, Hong Kong, SAR, Hong Kong

e-mail: kaili.c.zhang@gmail.com referred to as higher education in terms of global perspective, while relatively few studies have examined this phenomenon at the secondary education level.

The Hong Kong Examinations and Assessment Authority (HKEAA) revealed a recent case in which a student made use of a mobile phone to access the internet for an answer during a public examination $(\mathrm{Ng} 2006)$. The case was determined to be not disturbing at all, with the authorities concluding that there was "no evidence of widespread cheating." From an educator's perspective, the student's attempt to cheat in the manner described might only be the tip of the iceberg, which would, therefore, call for great concern.

A recent survey (Sing Tao 2008) conducted on more than 3,000 Primary Four to Form Seven students reaffirmed that the prevalence of cheating among secondary students is worthy of attention, given that more than one in five students admitted cheating on examinations. To make matters worse, more than half of the participants would not report cheating even if they had witnessed it. Students' reluctance to report peer cheating indicated the possibility of underestimation of cheating rates among students.

These findings were consistent with the results of the survey conducted by the Hong Kong Women Teachers' Organization (Sing Tao 2008). They produced further evidence that $30 \%$ of students had developed a habit of copying homework from their peers if they had forgotten to do it. All of the above implies a need to explore the motives of cheating among secondary students.

The significance of conducting research on the motives of cheating lies in the negative impact of academic dishonesty. From a pragmatic perspective, cheating undermines the use of assessment data as both an indicator of student learning and a source of feedback to teachers for instructional planning (Anderman and Murdock 2007). In 
terms of a social perspective, "the cheating learned or reinforced in school settings is related to a person's subsequent occupational or civic performance"; in short, it is "habit forming" (Cizek 2003, p. 36). The consequence of cheating for the entire education system was suggested by Cizek as an "erosion of the respect, trust, sense of community, and even student motivation for learning" (p. 36).

The magnitude of the problem of academic dishonesty has raised public concern over this issue. As a response to the growing concern about ethics in education from parents and the general public, the newly implemented School Based Assessment in Hong Kong was "designed in ways that students' work is to be done in class under direct teacher supervision" (Education Bureau 2007). This approach indicates the awareness and determination of the education sector to combat cheating among secondary school students under the new curriculum system.

The growing recognition of academic dishonesty as a major cross-cultural problem urges educators and researchers to examine various aspects of academic dishonesty (e.g., Murdock and Anderman 2006) . The primary purpose of these studies was to determine the prevalence and range of the problem, institutional and student demographic characteristics associated with cheating, and reasons that students give for cheating. This purpose also ultimately has implications on ways that cheating can be prevented.

\section{Contextual variables}

Peer attitude towards academic dishonesty

The relationship between students' beliefs about their peers' attitudes towards cheating, and the likelihood of their engagement in dishonest behaviours was thoroughly explored by Anderman and Murdock (2007). They found that students with a perceived belief that their peers condemned academic dishonesty were less likely to cheat. This result led to their conclusion that "peer disapproval is the most important determinant of changes in cheating behaviour" (p. 111).

Despite the fact that peer disapproval might deter cheating, Jendrek (1992) found students rather reluctant to express their disapproval of cheating, evidenced by their unwillingness to report the incident to authority figures or to stop the cheaters. The reluctance to report peer cheating is further elaborated in the next section. On one hand, expressing disapproval about academic dishonesty might deter it; on the other hand, peer attitudes towards the acceptability of academic dishonesty were dependent upon the perceived peer "culture of academic dishonesty." Given the interrelated nature between peer attitudes and cheating behaviour, Whitley and Spiegel (2002) postulated that students have the capacity to help prevent academic dishonesty by expressing disapproval of it.

\section{Peer reporting}

Numerous studies investigate students' reluctance to report witnessed cheating behaviour and the reasons behind it (Jendrek 1992; McCabe et al. 2001). In Jendrek's study, it was found that $61 \%$ of the sample reported observing some form of cheating, but that $55 \%$ said they ignored it. Although an additional 39\% said that they discussed the incident with students other than the cheater, only $5 \%$ said they told the cheater that they disapproved of the behaviour. The majority of the participants reported a "feeling of anger or disgust but nonetheless made no report", while the rest expressed an indifferent emotional response towards the cheating incident. A variety of reasons were given for not reporting cheaters, ranging from responses like "none of my business" to fear of making an enemy and even conflicting demands of loyalty posed by friendship if the cheater was a friend (McCabe et al.).

An explanation was put forward by Treviño and Victor (1991) for understanding the phenomenon that students had no intention of confronting their peers about their dishonesty and informing an authority figure about what was occurring. They proposed that "groups tend to create norms that support in-group loyalty." These norms were significant in ensuring "group cohesiveness and fostering feelings of security." Under these norms, peer reporting represented "a violation of group norms regarding loyalty which was likely for the peer reporters to face condemnation and even expulsion from the group" (p. 56). Other possibilities for this lack of action include unwillingness to undergo an emotional confrontation, and fear of retaliation by the cheaters, and even of being accused of harassment.

\section{Peer cheating}

Here, peer cheating represents both perceived norm of cheating and actual cheating rates. In understanding the influence of peer behaviour on cheating, Jordan (2001) conducted research and his studies suggest that more cheaters than non-cheaters believe that more students engage in cheating behaviours. These findings were consistent with previous research on the importance of peer norms for understanding, and perhaps influencing cheating behaviour (Whitley 1998).

Several explanations for the apparent gap between cheaters and non-cheaters' estimation of cheating rates have been suggested by researchers. First, the higher estimation by cheaters implies their attempts at preserving their self-image known as the false consensus effect. The 
perceived cheating culture is a reflection that students look to their peers for cues as to what behaviours are normative at their institutions (McCabe et al. 2001). This finding can be explained by the Social Comparison Theory, which proposes that people often look to others to validate their own attitudes and beliefs.

The more the cheaters believed the rest were cheaters, the more they believed cheating was acceptable, which constituted the peer pressure to cheat. The explanation was consistent with the study by McCabe et al. (2001) which found peer behaviour to be an important influence on academic dishonesty. As a result of being in a social context where cheating is acceptable, cheaters are more likely to learn the behaviour. Such relationships are supported by the Social Learning Theory (Bandura 1986) which emphasises that much of deviant behaviour is learned through the influence of example.

\section{Psychological variable}

\section{Self-efficacy}

Several correlational studies have directly examined selfefficacy beliefs in relation to cheating behaviour. For example, Murdock et al. (2001) reported an inverse relation between cheating and academic self-efficacy for middle school students, after controlling for personal goals, classroom goal structures, and other aspects of the classroom environment. Similar correlations between self-efficacy and cheating have been reported in college samples (Finn and Frone 2004). Other studies have linked cheating to various emotional arousals like fear of failure, test anxiety and doubt about one's performance (Anderman and Murdock 2007), all of which serve as low-efficacy cues.

The inverse relationship between academic efficacy and cheating was explained by Murdock et al. (2001) who proposed that "doubting one's ability to bring about a desired result might lead to reliance on other strategies for success", specifically referring to cheating. According to Murdock, low-efficacy breeds high avoidance, whereas self-efficacious students undertake difficult and challenging tasks more readily. By avoiding the task, cheaters succeed in guarding themselves against being in a situation that can potentially overwhelm, stress, confuse, frustrate and embarrass them.

In addition, there is no doubt that most research on academic dishonesty has been confined to an American context which raises concern as to whether the findings are applicable and comparable to a local context. At the same time, the Hong Kong Education sector has made limited attempts in addressing the increasing spread of academic dishonesty. Apparently, the significance of academic integrity has not been properly acknowledged and thus, research on cheating in Hong Kong does not exist.

Taking into account that such studies are lacking, the present paper examines cheating in classroom-based assessment modes, frequently referred to as examinations, in-class tests and homework, all of which are traditionally considered as fair forms of assessment in Hong Kong secondary schools. The purpose of the study is threefold: first, to determine the effect of peer attitudes and behaviour on the likelihood of cheating; secondly, to establish the significance of self-efficacy in promoting academic integrity; lastly, to ascertain effective ways of deterring academic dishonesty.

The primary questions this research addresses are as follows: (a) How does the level of self-efficacy affect engagement in cheating? (b) How does peer influence affect the likelihood of cheating?

The following definitions of pertinent terms are established for consistent reference and understanding throughout this research. Academic dishonesty refers to "all forms of cheating on tests or other academic assignments and plagiarism" (Anderman and Murdock 2007, p. 83). Cheating refers to "any action that violates the established rules governing the administration of a test or the completion of an assignment; any behaviour that gives one student an unfair advantage over other students on a test or assignment; or any action that decreases the accuracy of the intended inferences arising from a student's performance on a test or assignment" (Cizek 2003, p. 3). Self-efficacy concerns a person's expectation or judgment of how well or how poorly he or she will cope with a situation, given the skills possessed and circumstances one faces (Bandura 1986; Zimmerman 2000). Peer influence refers to the effect peers have on each others' behaviours.

The following assumptions were made as foundational to the investigation into the problem: (a) The survey participants will respond honestly and thoughtfully about their behaviours and beliefs concerning academic dishonesty. (b) A self-reporting survey of students is a reliable method for understanding the frequency and types of engagement in academic dishonesty of students and their peers. (c) Collection and analysis of survey data are reliable methods for conducting qualitative research.

\section{Methodology}

This research project studied cheating behaviours in traditional classroom contexts employing examinations and homework as assessment instruments of students' ability, as self-reported by secondary students. The above-mentioned purpose fits into the category of explanatory studies which are designed "to explain the forces causing the 
phenomenon in question and to identify plausible causal networks shaping the phenomenon" (Marshall and Rossman 1995, p. 138). Thus, the explanatory nature of this particular study incorporated the use of survey analysis rather than direct observation, which is comparatively inaccurate in inferring cheating behaviour. This study implemented qualitative methodology which provides for an in-depth study of "things in their natural settings" (Denzin and Lincoln 2000, p. 18) and was conducted using the questionnaire methodology, which follows the accepted practices in educational research and proves to be the most common and effective tool for collecting sensitive data, including cheating.

Based upon previous similar studies (Lathrop and Foss 2005; McCabe 1992), the questionnaire (Appendix 1) was designed by the researchers to elicit responses to questions about demographics, involvement in academic dishonesty in take-home assignments and examinations and to provide elaborate answers on reasons for academic dishonesty.

\section{Instrument}

A survey was generated to address the research questions. The components of the survey aimed at determining the demographic characteristics of the population, the extent of engagement in academic dishonesty, perception of influence of psychological and contextual factors on cheating, self-efficacy and peer influence. The section on demographic characteristics was followed immediately by a list of cheating behaviours which were selected based on definitions from relevant literature (e.g., McCabe 2001; McCabe and Treviño 1993). The majority of the questions were open-ended, which served to maximise participants' elaboration on domains of self-efficacy and peer influence, including perceived difficulty of given task and level of confidence, as well as peer attitudes and behaviours. In interpreting the data, only responses relevant to the particular questions were extracted and the rest were eliminated to maintain consistency.

\section{Participants}

The data for this research were derived from questionnaire responses of secondary school students who were representative of local Band 2 school students. The researchers chose this particular Band 2 school because it lacked comprehensive detection of students' academic dishonesty. The research project was expected to determine strategies for detecting and preventing cheating based upon students' responses.

Participants were of both junior and senior grades and considered to be in the top $20 \%$ in terms of academic achievement within their respective forms. A sample of
100 students was selected based on the suitability of population for qualitative research, in which only a small number of participants are recruited to contribute to a study. Using a small sample also allowed the researchers to study each participant's words and meanings in greater detail in order to produce conclusions which are rich in thick description (Lincoln and Guba 1985). All participants were contacted as a group in order to inform them of the purpose of the study, request their participation, assure their confidentiality and obtain informed consent.

The participants included 92 students of whom female and male were coincidently balanced in ratios of $50 \%$. Of the 92 students who participated in the research, junior students made up $42 \%$ while $58 \%$ were senior students. This sample was fairly representative of the secondary school population in terms of studies, gender and subjects taken.

Survey administration and data collection procedures

The procedure for administration of the survey and collection of the data was submitted to the principal and the lead teachers of the corresponding classes for approval and coordination. Upon approval of the research project, the questionnaire instrument was distributed to participants in person in an English lesson during which students were assumed to use English as a means as a means of communication more readily. A verbal and written notification regarding the administration of the survey, the collection and analysis of the data, possible uses of the data and most important of all, the consent form assuring anonymity was given to them prior to the completion of the questionnaire. As participants displayed an elementary level of spoken and written English but an advanced level of Chinese, direct translation of questionnaire items into Chinese was made available and delivered upon the request of participants. The translation of questionnaire times accounted for $40 \%$ of the total items, in other words, 6 of 16 questions were translated into Chinese. Students were required to hand the questionnaire in directly to the teacher-in-charge to avoid any unnecessary disclosure or loss of data. The privacy of students' responses was ensured by placing the questionnaires in an envelope before handing them to students so that their answer would not be seen by the teacher-in charge.

The questionnaire was made available for three weeks during the second semester to students of different grades who were impartially and randomly selected. During the collection period, 100 questionnaire submissions were recorded. Each submission was then carefully examined by the researchers and questionnaires that were all blank and with apparent standardised answers were deemed invalid as no apparent mental processing of question times were 
observed. Standardised answers were characterised by the same set of responses throughout the questionnaire. The more apparent case involved the use of the phrase "I don't know" for the entire questionnaire. In this research, standardised answers made up $40 \%$ of the total questionnaire responses. The response rate based on the total questionnaires distributed was $92 \%$ with the remainder regarded as invalid.

\section{Findings}

Data analysis was based on the transcribed version of participants' responses to the questionnaire. By employing the grounded theory proposed by Glaser and Strauss (1967) in data analysis of qualitative methodology, the transcribed data was first categorised and labelled, in the process known as open coding. This allowed for further generation of concepts and identification of relationships among components in an axial coding stage. Finally, the data were categorised into conceptual domains of self-efficacy and peer influence including level of self-efficacy, peer reporting, observation of peer cheating and peer disapproval. The processes of open and axial coding were merged because of the need to discover theory from data as grounded theory focuses on theory confirmation, which is a process of testing hypotheses developed from previous theories, rather than on theory generation (Glaser and Strauss 1967). Finally, the results of this study were reported in narrative statements which encapsulated the group's construction of academic dishonesty. These statements include rich descriptions of the categories that evolved from the data analysis, a compilation of detailed information about participants' meaning-making and quotes as supportive evidence.

\section{Reported academic dishonesty rates}

The data analysis was based on the responses to different forms of academic dishonesty that have been identified in literature. Students responded by putting a tick next to the cheating behaviour in which they had engaged. The types of cheating behaviours listed in the questionnaire were further sub-divided into academic dishonesty during tests, academic dishonesty on out-of-class assignments and academic dishonesty in high-stake examinations, all of which reflected a continuum of behaviours that ranged from least to most server.

As hypothesised, from the responses representing the general population of secondary school students, the overall data revealed the prevalence and extent of academic dishonesty in secondary schools. Only $6 \%$ of the students reported that they had never engaged in any of the
9 listed cheating behaviours (please refer to Appendix 1, question 3); whereas, over $90 \%$ admitted to at least one incident of cheating. The extent to which academic dishonesty occurred might be shocking but by no means should it be overlooked by educators.

From all the cheating behaviour in which participants engaged, the highest rate of academic dishonesty was found in out-of-class assignments. Cheating on a test came next, while the least reported cheating behaviour was found in high-stakes examinations. The significant gap between cheating in out-of-class assignments and examinations might be explained by the perception of the severity of assessment modes. Apparently, high-stakes examinations as a form of assessment was perceived to be an important and reliable tool for assessment, which drove students to be responsible for their own behaviour.

\section{Perception of situational experiences}

Participants' perception of situational experiences was assessed by eliciting their personal attitudes and views towards specific situational experiences, in this case, highstakes examinations, tests and assignments. From the data collected on Questions 7 and 8, perspectives on situational experiences were largely sub-divided into three emotional extremes: like, neutral and dislike.

Illustrated in Fig. 2 in Appendix 2, over $80 \%$ of the participants considered taking examination to be stressful and even showed a positive attitude. One senior student, who reported rare engagement in academic dishonesty, described examinations as follows:

Examinations reinforce acquired knowledge and provide an opportunity for us to revise. I enjoy this a lot.

A student similarly described examinations as a constructive experience:

Examinations are a way to increase knowledge and, to me, the stress serves as a source of motivation.

Among the $80 \%$ of participants (see Fig. 2 in Appendix 2) who considered taking examinations as stressful, the majority reported a sense of pressure from three primary groups: parents, self and peers. One senior student highlighted how parental pressure in particular reinforced his cheating behaviour:

I experience immense parental pressure to be academically successful to the extent that I never have enough time to sleep and this leads to poor quality of sleeping which seriously affected my attentiveness.

Another female student explained how she perceived peers as a source of pressure to succeed: 
Even though I put effort into revising, I still cannot perform better academically than my peers and I often feel that I lag behind.

Remarks in Questions 7 and 8, as shown in Figs. 4 and 5 in Appendix 2, reflected that $57 \%$ of participants displayed a negative view of homework, similar to that of $49 \%$ of participants who disliked examinations. A senior student, whose responses represented the comments from both "dislike" groups, viewed both homework and high-stakes examinations as necessary and constructive experiences:

The more we work hard on homework, the more it is useful for us in acquiring knowledge. Simultaneously, examinations are a chance for us to assess our acquired knowledge in different domains.

A minority group of $28 \%$ students held an indifferent attitude towards high-stakes examinations. One student noted:

These assessments come with our responsibility as students and therefore, are unavoidable.

However, a large proportion of participants (49\%) indicated a feeling of dislike towards high-stakes examinations by describing them as worthless and time-consuming. One student gave a seemingly sensible argument for the distressing nature of this type of assessments:

The examination itself is ridiculous and is even a torture for us, given that all that matters is marks and grading. The results exert only a labelling effect on students, which in turn becomes a source of pressure.

\section{Self-efficacy}

The level of self-efficacy was determined by having students judge their overall academic performance and their level of confidence towards a specific task. Consistent with past research on the role of self-efficacy in the promotion of cheating (Cizek 1999; Murdock et al. 2001), we found that self-efficacy is correlated with cheating, which was also further supported by Finn and Frone (2004). These researchers maintained that cheating was higher for students with lower levels of self-efficacy regardless of performance level. This finding was evident in $95 \%$ of the participants, shown in Fig. 3. When asked whether they would cheat in cases where they had more confidence in succeeding, over $90 \%$ of students responded "No" to the question. One student gave a thorough explanation of her choice:

Having more confidence means I have the knowledge required and cheating in this case is not necessary.
Moreover, the others might have inaccurate answers, so in this case, cheating is not a reliable ways to succeed.

Among the participants who responded "No" to the question, 5\% displayed consistent honesty regardless of any contextual or psychological factors, viewing cheating as:

an unfair means that exploits the rights of the rest, while for those cheaters satisfaction is not likely to arise because they are not using their own strengths.

For the rest of the participants indicating "Yes" to Question 6, cheating seemed to be an effective way to ensure a passing, or even a good, grade. The pressure to succeed academically might have been so overwhelming that they chose to cheat despite having confidence. The way an efficacious participant related her motive to cheat within the role of the examination system was noteworthy:

The school values merely the results but not the learning process. So, I don't care how I achieve the desired result even if I cheat. To me, only the outcome itself matters.

Since the level of self-efficacy depends on the difficulty of a particular task (Murdock et al. 2001), participants were asked to judge the nature of high-stakes examinations and homework in questionnaire items 7 and 8 in order to determine their levels of self-efficacy. From the responses indicated on questionnaire items 7 and 8, about $50 \%$ of participants perceived both of the given tasks as challenging, which leads to the implication that they had lower self-efficacy. This belief was directly predictive of the choice of activities undertaken. This study revealed that inefficacious students undertook challenging tasks less readily than did efficacious students. In terms of engagement in academic dishonesty, the $50 \%$ of inefficacious participants were more prone to cheating.

Peer influence

\section{Peer reporting}

Not surprisingly, $94 \%$ of participants witnessed peer cheating in class as noted in Fig. 6. Yet, despite the pervasiveness of academic dishonesty, 79\% of participants made no attempts to prevent it. When asked how they responded to peer cheating, the norm of nondisclosure prevailed, with over half of the participants deciding to maintain silence. A senior student discussed the possible undesirable consequences of reporting cheating to teachers or confronting the cheaters:

Informing an authority figure about who cheats is likely to result in being hated by close friends and making enemies. This act interferes with my interpersonal relationships. 
To those who viewed loyalty as the primary criterion of being a member of the peer group, anyone who reported cheating was regarded as a "squealer." To make matters worse, loyalty to friends served as an excuse for $14 \%$ of participants to comply with cheaters:

Report of peer cheating is likely to result in being despised or disliked by friends and being regarded as squealers. Usually, I follow what the cheaters do in order to be friends with them.

Some reporters of peer cheating, comprising $3 \%$ of the total participants, touched upon loyalty when they disclosed cheaters' dishonest acts. Only in cases where they regarded cheaters as enemies was loyal to friends not applicable. Therefore, reporting of academic dishonesty was evident in those who viewed cheaters as enemies. Unlike those who viewed cheaters as enemies, other reporters of cheating showed genuine signs of belief in justice and academic integrity by requesting the cheaters to stop. A female student explained what motivated her to discourage cheaters from cheating:

When I see others cheat, it frustrates me and those who spend time revising. I will definitely stop cheaters in order to be fair to those who rely on their own efforts.

It was obvious from the participants that cheating was widespread in the classroom. Even though the prevalence rate of cheating was an alarming 90\%, it was hardly in line with the participants' reporting rate at only 3\%. Treviño and Victor (1991, p. 54) offered a meaningful explanation for why resistance of peer reporting exists:

Peer reporting is generally discouraged within groups, because groups tend to create norms that support in-group loyalty. These norms can be highly adaptive, ensuring group cohesiveness and fostering feelings of security.

When any group member engaged in misconduct, other group members preferred to handle the situation within the group and often reacted negatively when members ventured outside the group to report the misconduct (Treviño and Victor 1991). The results of the data analysis show uniformity with their elaboration on norms discouraging peer reporting. The majority of participants chose either to persuade the cheater to stop or to ignore the cheating behaviour despite revealing intense negative emotions towards the dishonest act. Obviously, under the influence of norms discouraging peer reporting, the negative attitudes and emotions experienced by potential peer reporters did not appear to play a role in deterring the cheaters from further misconduct.

\section{Observation of peer cheating}

Another issue that surfaced was the influence of observational peer cheating as shown in Figs. 7 and 8 in Appendix 2. In this study, one half of the participants claimed that they would not cheat even if they knew some of their classmates cheated. Reasons ranged from honest and confident attitudes to unwillingness to be part of the cheater group. A senior student exhibited a mature and sensible rationale for maintaining academic honesty:

blindly following what the cheaters do cause guilt; I don't think I will have a sense of satisfaction even if I succeed with cheating.

A minor $30 \%$ of students claimed that they would follow the norm of cheating in view that the assessment system was no longer a fair instrument.

Witnessing peer cheating reinforces the motives to cheat because in a company of peers, it seems to me that it is less risky to be punished. Most importantly, the assessment system is not fair anyway.

Under the influence of peer cheating, the motives to cheat depended on both personal factors such as the level of familiarity towards examinations, and external factors such as the importance of assessment modes and the likelihood of being caught cheating.

Given the hypothetical situation that no one cheated in class, $92 \%$ of participants reported that they would not cheat, citing a number of factors such as fear of authority, lack of a "leader", fairness to others and preservation of the group image. One student explained her choice in terms of group normative behaviour and observational learning:

If none of your friends cheat, the only way to be part of the group is by following the norm of honesty so that a positive image as a group can be maintained. When no one cheats, it simply means that it is risky to cheat.

From data analysis on influence of peer behaviour as reflected in Questions 10 and 11, two distinctive behaviours were observed among participants when witnessing peer cheating. This distinction was understood with regard to the intimacy of the relationship between the witnesses and cheaters. As exemplified by the Differential Association Theory, the witnesses' acquisition of deviant behaviour was dependent upon their association with the cheaters. Therefore, in this research study, participants who indicated the decision of following the norm of cheating were likely to have closer association with cheaters than those who decided not to cheat.

An alternative justification for the above distinction was put forward by McCabe and Treviño (1993), who proposed incorporating rewards of cheating as an essential element preceding the learning of cheating. They claimed that peer 
behaviour was not simply an important influence on academic dishonesty, but specifically a risk factor for learning to cheat. Hence, merely witnessing cheating is not enough to cause witnesses to cheat. In this study, $58 \%$ of participants refused to cheat even while observing peer cheating for the reason of a perceived lack of reward. These "witnesses" might not yet have been aware of the "reward" of cheating, which was represented by cheaters succeeded in cheating without being exposed. Therefore, they instead made a decision not to take the risk of being reported as cheaters.

Coincidentally, the rationale given by those who imitated the cheating behaviour of their peers showed consistency with the above theoretical explanation. As shown in this study, participants attributed their peer imitation of cheating to the success brought about by cheating, such as getting a good grade and escaping unnoticed. As a conclusion, seeing one's friend cheat successfully increased the tendency of the observers to behave in similar ways. The above theories regarding association between peer behaviour and cheating imply that having immediate negative consequences for cheating is of utmost importance in creating a culture of academic integrity.

\section{Peer disapproval}

To account for how peers exert influence on cheating and how peers can deter cheating, the perception on those who cheat and who do not cheat are worth considering. Extreme and contradictory views towards non-cheaters were revealed in questionnaire item 13 and Fig. 10 in Appendix 2. Of the participants, $76 \%$ described non-cheaters as smart, confident and responsible students. On the contrary, $12 \%$ of the participants, the majority of whom were cheaters themselves, expressed a completely conflicting perception, seeing the non-cheaters as being hypocrites and inflexible. Their negative perception stemmed from the motivation to despise non-cheaters who displayed "deviant behaviour" from the majority.

Attitudes towards cheaters were asked about in Question 15 and Fig. 12 in Appendix 2. To our surprise, merely 29\% of participants had a negative attitude towards cheaters who were being portrayed as lazy and hateful. For the rest of the participants, $41 \%$ had a neutral attitude, as reflected by the response of the following participant:

I don't care whether and why they cheat as long as their behaviour does not affect me.

Interestingly, $30 \%$ of the participants displayed positive feelings towards cheaters, regarding them as smart and brave. Most importantly, the role of the cheater as a significant member of the group was particularly highlighted by one participant: "I feel proud of the cheaters and I consider them as important members of our group."

\section{Summary and implications for educators}

The purpose of this study was to determine the role of selfefficacy and peer influence on the behaviour of cheating. Though the causal relationship is yet to be determined, findings suggest that self-efficacy is correlated with academic dishonesty. These findings also serve to remind educators of their responsibility to employ strategies to increase self-efficacy, such as setting reasonable levels of acceptable performance and rewarding students at all achievement levels for hard work and learning (Finn and Frone 2004). These strategies are believed to reduce cheating among students who perceive themselves to be at risk for failure. However, more research is needed in order to further explore the possible cause-and-effect relationship between self-efficacy and academic dishonesty.

Another purpose of this research was to determine how peers exert influence on cheating behaviour. The data reflected that peers are clearly in a position to detect academic dishonesty in traditional classroom contexts. Therefore, educators should create norms that encourage peer reporting by adopting an official code of reporting, often referred to globally as an honour-code environment (McCabe et al. 2001). In these honour-code environments, role responsibility for peer reporting is higher and the culture of academic integrity is stronger. In addition, in honour-code environments, one can expect greater commitment to and involvement in the academic environment along with a greater acceptance of moral validity in norms against cheating behaviours.

This research emphasised that the belief of self-efficacy is one of the determinants of cheating behaviour. This practical relationship suggests that educators should build and strengthen students' self-efficacy beliefs. As students face challenging and difficult circumstances, they rely on external information to inform their sense of self-efficacy (Schunk 1991). Teachers are advised to devote their attention to these sources of self-efficacy information, using verbal persuasion to assure students' coping ability. Self-efficacy is related to peer influence in terms of vicarious experience in which students closely observe how well their peers are coping. Therefore, the manner in which students handle difficult and potentially overwhelming situations serves as a model for their peers. This chain effect deserves attention of educators who must strengthen struggling learners' self-efficacy by rewarding positive effort and persistence.

Given the reluctance of peer reporting, McCabe et al. (2001) proposed creating normative counter-pressures which promote peer reporting of cheating behaviour. Building upon role responsibility, it appears that peer reporting is likely to occur where peer reporting is expected as part of one's role responsibility. Simply speaking, the 
greater the role responsibility a student is perceived to have, the higher the inclination to report cheating behaviour among peers. In addition, the clear definition of peer reporting as part of one's role guaranteed students' acknowledgement of the explicit guidance regarding the expected and appropriate behaviour of reporting an act of cheating.

With the existence of role responsibility, the practice of peer reporting is anticipated to override the practice of the code of silence. Therefore, in deterring academic dishonesty, educators must foster a sense of responsibility among students by encouraging the report of any violation of rules. This will serve as a counter-pressure to the strong norms supporting in-group loyalty.

The role of peer behaviour in the encouragement of cheating is still undetermined. Since a balanced ratio was observed between those who followed the norm of cheating and those who remained academically honest regardless of peer behaviour. The results reflect, to a certain degree, the compatibility between Social Learning Theory and Differential Association Theory. These theories emphasise that much of human behaviour is learned through the influence of example (Bandura 1986) and that deviant behaviour is influenced by close association with others involved in deviant behaviour. In other words, dishonesty is learned rather than innate.

\section{Recommendations for further research}

As with other studies, this study acknowledges some limitations, including the fact that the population of this study was limited to students from a local Band 2 school with Chinese as a medium of instruction. With such a limited sampling, the validity of generalising the results for the whole secondary school population is undoubtedly low. In addition, reliance on self-report in this study may be an important limitation.

This research aimed at drawing educators' attention to possible ways of tackling the widespread academic dishonesty and stimulating further, in-depth research into the issue of academic dishonesty. As mentioned in the limitations of this study, the findings were limited to the population enrolled in that particular secondary school in determining the role of self-efficacy and peer influence in promoting a culture of academic integrity. It would be advantageous for researchers to extend these findings by including randomly selected samples from various bandings of local secondary schools so as to better understand the interplay between these domains and academic dishonesty, and to promote a culture of academic honesty.

Although research into the role of self-efficacy and peer influence has been widely pursued, comprehensive study which incorporates contextual variables such as classroom settings, institutes' attitudes and policy towards cheating is still overlooked and thus, recommended for further research. Evidence from previous research suggests that quantitative research has taken precedence over qualitative study. Therefore, it is recommended that qualitative epistemology be conducted in order to gain a deeper understanding of the reasons behind student perceptions.

Open Access This article is distributed under the terms of the Creative Commons Attribution Noncommercial License which permits any noncommercial use, distribution, and reproduction in any medium, provided the original author(s) and source are credited.

\section{Appendix 1: Student questionnaire instrument: motives of cheating among secondary school students questionnaire}

1. What is your current level of study?

2. What is your gender?

3. Have you engaged in the following behaviour which is considered as cheating?

Copying from another student during a test

Cheating on a test in any other way

Copying materials (e.g., worksheets) and handing them in as your own work

Working together on homework when the teacher asked for individual work

$\square$ Handing in work done by others

Changing an answer after an exam was graded and reporting it as a scoring error

$\square$ Giving exam/quiz questions to students who would take the exam/quiz later

Allowing someone to copy during an exam

Intentionally looking at another student's answer and keeping your answer if it is the same

4. What is your general expectation of results in examinations?

5. Do you consider taking examinations as stressful and why?

6. If you have more confidence in getting a good grade, would you cheat and why?

7. How do you like homework?

8. What do you think of examinations?

9. Have you observed someone cheating in your class? What would you do in this case and why?

10. If you know some of your classmates cheat, will you cheat and why?

11. If no one in your class cheats, will you cheat and why? 
12. Would you report to the teacher if you see someone cheating? Why?

13. How will you view those who do not cheat?

14. Have your friends copied your homework? Why do you allow them to do so?

15. How do your friends feel about you if you cheat?

16. Is there anything that you would like to let us know?
THANK YOU FOR COMPLETING OUR QUESTIONNAIRE!

Appendix 2: Figures of data analysis from questionnaire items

See Figs. 1, 2, 3, 4, 5, 6, 7, 8, 9, 10, 11, 12.
Fig. 1 Questionnaire item 4:

What is your general expectation of results in examination?

\begin{tabular}{|l|l|}
\hline Responses & No. of students \\
\hline Yes & 76 \\
\hline No & 9 \\
\hline It depends & 7 \\
\hline
\end{tabular}

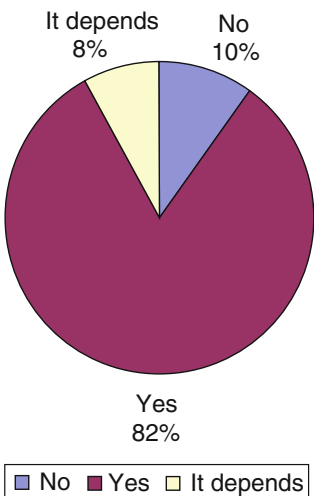

Fig. 2 Questionnaire item 5: Do you consider taking examinations as stressful and why?

\begin{tabular}{|l|l|}
\hline Responses & No. of students \\
\hline Yes & 5 \\
\hline No & 87 \\
\hline
\end{tabular}

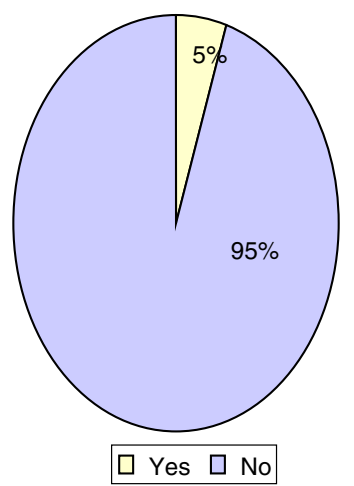

Fig. 3 Questionnaire item 6: If you have more confidence in getting a good grade, would you cheat and why?

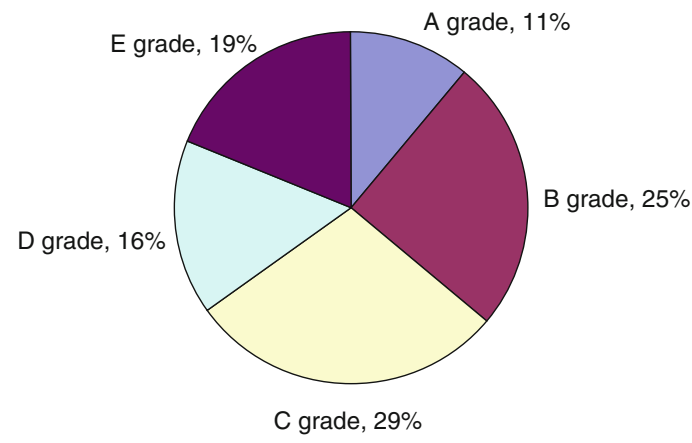

$\square$ A grade $\square$ C grade $\square$ E grade

$\square$ B grade $\square$ D grade

\begin{tabular}{|l|l|}
\hline Responses & No. of students \\
\hline Positive & 10 \\
\hline Neutral & 30 \\
\hline Negative & 52 \\
\hline
\end{tabular}

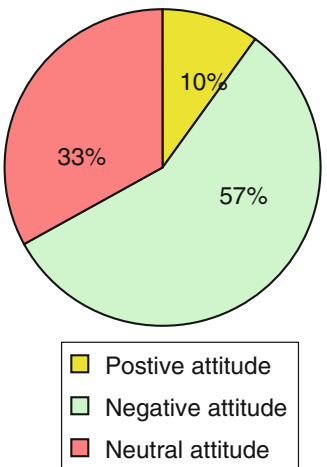

Fig. 4 Questionnaire item 7: How do you like homework?

\begin{tabular}{|l|l|}
\hline Responses & No. of students \\
\hline Positive & 21 \\
\hline Neutral & 26 \\
\hline Negative & 45 \\
\hline
\end{tabular}

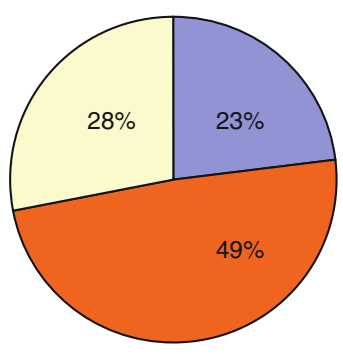

Positive attitude

Negative attitude

Neutral attitude

Fig. 5 Questionnaire item 8: What do you think of examinations? 


\begin{tabular}{|l|l|}
\hline Responses & $\begin{array}{l}\text { No. of } \\
\text { students }\end{array}$ \\
\hline No & 5 \\
\hline $\begin{array}{l}\text { Yes; report to } \\
\text { teachers }\end{array}$ & 2 \\
\hline Yes; keep silence & 60 \\
\hline $\begin{array}{l}\text { Yes; stop the } \\
\text { cheaters }\end{array}$ & 11 \\
\hline Yes; cheat together & 14 \\
\hline
\end{tabular}

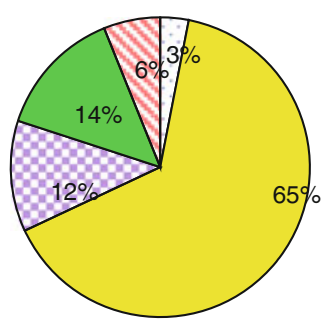

$\square$ Yes- report to teachers $\square$ Yes- keep silence $\square$ Yes- stop the cheater $\square$ Yes- cheat together $\checkmark$ No

Fig. 6 Questionnaire item 9: Have you observed someone cheating in your class? What would you do in this case and why?

\begin{tabular}{|l|l|}
\hline Responses & No. of students \\
\hline Yes & 27 \\
\hline No & 53 \\
\hline It depends & 12 \\
\hline
\end{tabular}
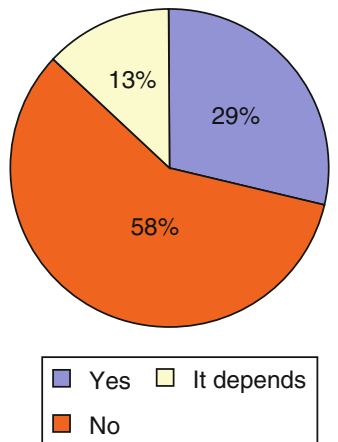

Fig. 7 Questionnaire item 10: If you know some of your classmates cheat, will you cheat and why?

\begin{tabular}{|l|l|}
\hline Responses & No. of students \\
\hline Yes & 3 \\
\hline No & 85 \\
\hline It depends & 4 \\
\hline
\end{tabular}

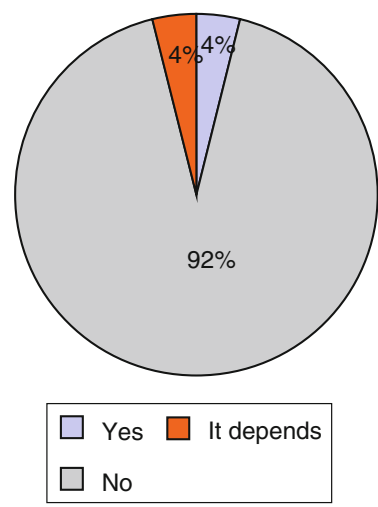

Fig. 8 Questionnaire item 11: If no one in your class cheats, will you cheat and why?

\begin{tabular}{|l|l|}
\hline Responses & No. of students \\
\hline Yes & 6 \\
\hline No & 82 \\
\hline It depends & 4 \\
\hline
\end{tabular}

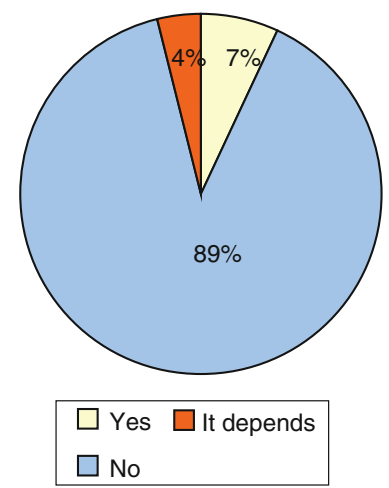

Fig. 9 Questionnaire item 12: Would you report to teacher if you see someone cheating? Why?
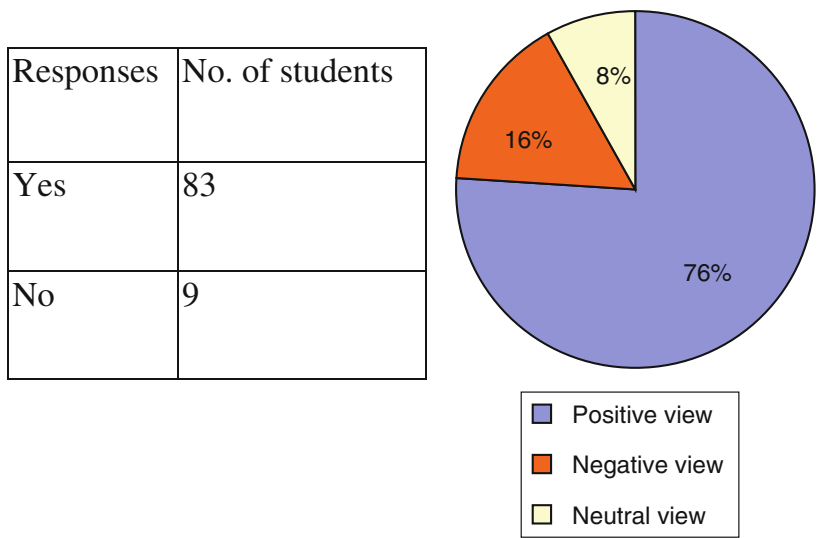

Fig. 10 Questionnaire item 13: How will you view those who do not cheat?

\begin{tabular}{|l|l|}
\hline Responses & No. of students \\
\hline Feelings of like & 27 \\
\hline $\begin{array}{l}\text { Indifferent } \\
\text { feeling }\end{array}$ & 38 \\
\hline $\begin{array}{l}\text { Feelings of } \\
\text { dislike }\end{array}$ & 27 \\
\hline
\end{tabular}

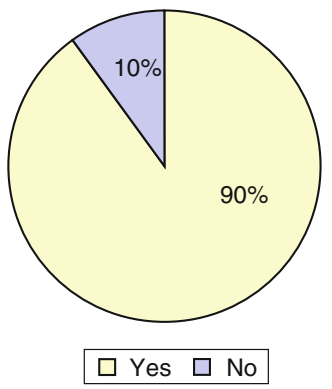

Fig. 11 Questionnaire item 14: Has your friend copied your homework? Why do you allow her to do so? 


\begin{tabular}{|l|l|}
\hline Responses & No. of students \\
\hline Positive & 74 \\
\hline Negative & 11 \\
\hline Neutral & 7 \\
\hline
\end{tabular}

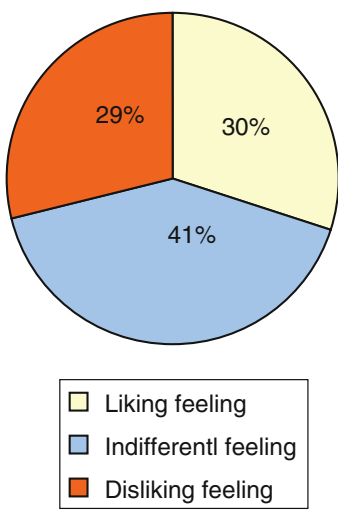

Fig. 12 Questionnaire item 15: How do your friends feel about you if you cheat?

\section{References}

Anderman, E. M., \& Murdock, T. B. (2007). Psychology of academic cheating. London: Elsevier Academic Press.

Bandura, A. (1986). Self-efficacy: The exercise of control. New York: W. H. Freeman.

Cizek, G. J. (1999). Cheating on tests: How to do it, detect it and prevent it. New Jersey: Lawrence Erlbaum Associates.

Cizek, G. J. (2003). Detecting and preventing classroom cheating: Promoting integrity in assessment. California: Corwin.

Denzin, N. K., \& Lincoln, Y. S. (2000). The discipline and practice of qualitative research. In N. K. Denzin \& Y. S. Lincoln (Eds.), Handbook of qualitative research (2nd ed., pp. 1-28). Thousand Oaks, CA: Sage.

Finn, K. V., \& Frone, M. R. (2004). Academic performance and cheating: Moderating role of school identification and selfefficacy. Journal of Educational Research, 97(3), 115-122.

Glaser, B. G., \& Strauss, A. L. (1967). The discovery of grounded theory: Strategies for qualitative research. London: Weidenfeld and Nicolson.

Jendrek, M. P. (1992). Students' reactions to academic dishonesty. Journal of College Student Development, 33, 260-273.

Jordan, A. E. (2001). College student cheating: The role of motivation, perceived norm, attitudes and knowledge of institutional policy. Ethics and Behavior, 11(3), 233-247.

Lathrop, A., \& Foss, K. (2005). Guiding students from cheating and plagiarism to honesty and integrity: Strategies for change. USA: Libraries Unlimited.
Lincoln, Y. S., \& Guba, E. G. (1985). Naturalistic inquiry. Newbury Park, CA: Sage.

Marshall, C., \& Rossman, G. B. (1995). Designing qualitative research (2nd ed.). Thousand Oaks, CA: Sage.

McCabe, D. L. (1992). The influence of situational ethics on cheating among college students. Sociological Inquiry, 62, 365-374.

McCabe, D. (2001). Cheating: Why students do it and how can we help them stop. American Educator, 25(4), 38-43.

McCabe, D. L., \& Treviño, L. (1993). Academic dishonesty: Honor codes and other contextual influences. Journal of Higher Education, 64, 522-538.

McCabe, D. L., Treviño, L. K., \& Butterfield, K. D. (2001). Dishonesty in academic environments: The influence of peer reporting requirements. The Journal of Higher Education, 72(1), $29-45$.

Murdock, T. B., \& Anderman, E. M. (2006). Motivational perspectives on student cheating: Toward an integrated model of academic dishonesty. Educational Psychologist, 41(3), 129-145.

Murdock, T. B., Hale, N. M., \& Weber, M. J. (2001). Predictors of cheating among early adolescents: Academic and social motivations. Contemporary Educational Psychology, 26, 96-115.

$\mathrm{Ng}$, T. (2006). Mobile used to get exam answers online. Retrieved May 20, 2006 from: http://www.chinadaily.com.cn/china/ 2006-05/20/content_595981.htm.

Schunk, D. (1991). Self efficacy and academic motivation. Educational Psychologist, 26, 207-231.

Sing Tao. (2008). Teachers blame parents for kids' moral decline. Retrieved September 22, 2008 from: http://edu.singtao.com/ engs/digest_details.asp?article_id=90\&catid=1.

Taylor, L., Pogrebin, M., \& Dodge, M. (2003). Advanced placement-Advanced pressures: Academic: Dishonesty among elite high school students. Educational Studies, 12, 403-421.

Treviño, L. K., \& Victor, B. (1991). Peer reporting of unethical behaviors: A social context perspectives. Academy of Management Journal, 35, 38-64.

Whitley, B. E. (1998). Factors associated with cheating among college students: A review. Research in Higher Education, 39, 235-274.

Whitley, B. E., \& Spiegel, P. K. (2002). Academic dishonesty: An educator's guide. New Jersey: Lawrence Erlbaum Associates.

Zimmerman, B. J. (2000). Self efficacy: An essential motive to learn. Contemporary Educational Psychology, 25, 82-91. 